\title{
THE EFFECTIVE ELECTRON-POSITRON POTENTIAL IN JELLIUM
}

\author{
H. Stachowiak, E. Boroński and J. Lach \\ W. Trzebiatowski Institute of Low Temperature and Structure Research \\ Polish Academy of Sciences, P.O.Box 937, 50-950 Wrocław 2, Poland
}

One of the methods of determining theoretical annihilation characteristics in real metals is the approximation called Bloch modified ladder approach. In this approach a Bethe-Goldstone type equation is solved with an effective electron-positron potential obtained previously for jellium of the corresponding electron density. If one wishes to include the dependence on the local electron density of the $\mathrm{e}^{+}-\mathrm{e}^{-}$effective potential in this formalism, it is necessary to know this potential for jellium, for metallic and above metallic densities. A review of different proposed $e^{+}-e^{-}$potentials is presented and their correctness is evaluated from the point of view of their application in a Bethe-Goldstone type formalism which is the jellium analogue of Bloch modified ladder approach.

PACS numbers: 78.70.Bj, 71.60.+z

\section{Introduction}

The purpose of this work is to study how successful different effective electron-positron potentials are in a Bethe-Goldstone (BG) formalism. A review of existing approaches in the theory of $\mathrm{e}^{+}-\mathrm{e}^{-}$interaction in metallic systems is presented in Ref. [1]. The BG-type approach proposed first by Kahana [2] leads to reliable results only if the potential used in the $B G$ equation is self-consistent $[3,4]$. Self-consistency, however, is difficult to obtain within the $B G$ formalism, so it is interesting to find out to what extent the potentials obtained in other approaches can be used successfully in the BG equation. In particular, we would like to know how the potential calculated using the approach called perturbed hypernetted-chain approximation (PHNC) $[5,6]$ works when applied in the BG equation. The BG formalism is particularly important from our point of view since it underlies one of the existing approaches to $\mathrm{e}^{+-} \mathrm{e}^{-}$interaction in real metals [1]. Indeed the formalism developed by Carbotte and Salvadori [7] and applied later extensively by Sormann under the name of Bloch modified ladder approach (BML) $[8]$ is the real metal analogue of the BG approach which in its original form applies only to jellium. The scattering of the state with momentum $p$ on the positron is described by the amplitudes $\chi_{p}^{n l}(k)$ which obey the equation

$$
\chi_{p}^{n l}(k)=1+\frac{1}{\Omega} \sum_{k^{\prime}} \chi_{p}^{n l}\left(k^{\prime}\right) \frac{\theta\left(k^{\prime}-k_{\mathrm{F}}\right)}{k^{\prime 2}+\left(p-k^{\prime}\right)^{2}+\Delta_{n l}} V_{\mathrm{s}}\left(k-k^{\prime}\right),
$$


where $\theta\left(k^{\prime}-k_{\mathrm{F}}\right)$ equals 1 for states $\mathrm{k}^{\prime}$ outside the Fermi surface and 0 otherwise (for details cf. [1] and the original papers). In Eq. (1) it is assumed that the potential of $\mathrm{e}^{+}-\mathrm{e}^{-}$interaction $V_{\mathrm{s}}$ is position independent. This assumption is physically incorrect since it assumes that only the electrons from the outer shell participate in the screening of the positron. The approximation underlying BML could be improved by allowing for a position dependence of the $\mathrm{e}^{+}-\mathrm{e}^{-}$potential of the interaction. In the case when the local density approximation is used (cf. [9], [10]), the local shape of the $\mathrm{e}^{+}-\mathrm{e}^{-}$potential should correspond to the potential calculated for jellium of density being equal to the local density of the electron gas in the real metal. In this way, the need appears for an $\mathrm{e}^{+}-\mathrm{e}^{-}$potential computed for all local electron densities occurring in a real metal, especially for high densities $\left(r_{\mathrm{s}}<2\right)$. No completely self-consistent potentials have been obtained for these densities within the BG formalism. Therefore, to these cases we will try to apply the potentials obtained self-consistently within other approaches, in particular the PHNC potential, the hypernetted-chain potential (HNC) in the form proposed by Gondzik and Stachowiak [11], the random phase potential (RPA) and the potential proposed by Jarlborg and Singh [12]. We are also interested how these potentials work at metallic densities when used in the BG equation, since self-consistent calculations have been performed within the BG formalism for very few values of $r_{\mathrm{s}}(2,4$ and 6$)[4,8]$.

\section{Results}

In this short contribution we can present only a small part of the results obtained in our calculations. Moreover, some more interesting aspects must still be investigated.
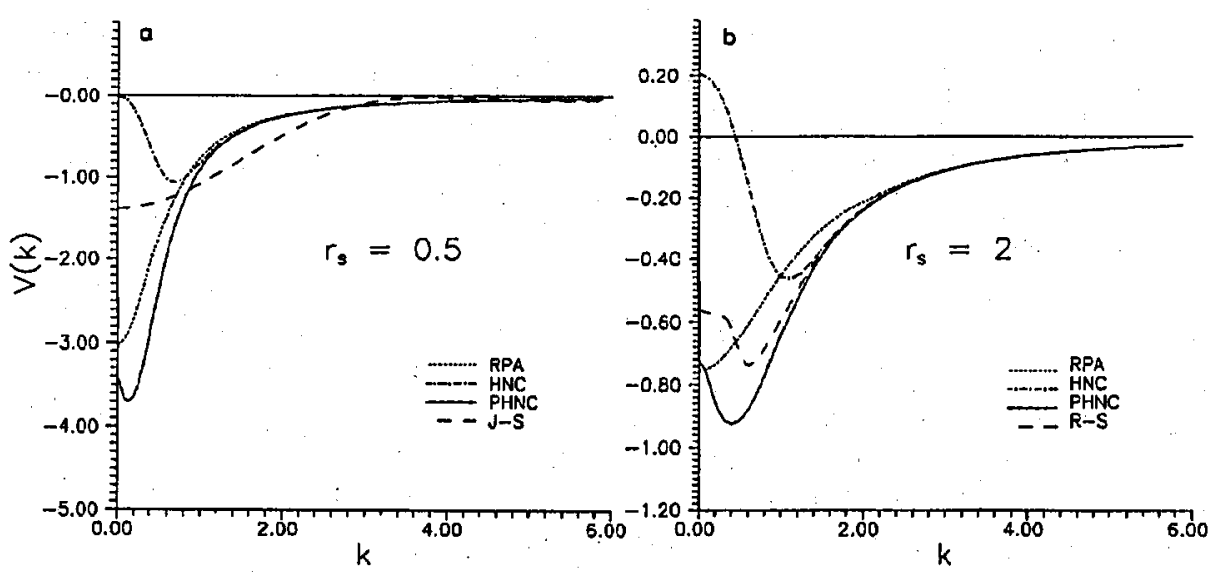

Fig. 1. Fourier coefficients $V(k)$ of different potentials for $r_{s}=0.5$ (a) and $r_{s}=2(b)$. Label R-S corresponds to the results of Ref. [4].

Some of the potentials used in our calculations are shown (in the momentum space) in Fig. 1 for $r_{s}=1$ and 2. We give here the formula for the Jarlborg-Singh 
(J-S) potential which has not been used frequently in the positron physics

$$
V(r)= \begin{cases}1 / r & r<r_{\mathrm{s}}^{\prime}, \\ 0 & r>r_{\mathrm{s}},\end{cases}
$$

where $r_{\mathrm{s}}^{\prime}=r_{\mathrm{s}}\left\{0.61\left[2 / 3+0.21\left(r_{\mathrm{s}} / 5\right)^{1 / 2}\right]\right\}^{1 / 2}$ and $V(r)$ is interpolated linearly between $r_{\mathrm{s}}^{\prime}$ and $r_{\mathrm{s}}$. The value of $r_{\mathrm{s}}^{\prime}$ is connected with the size of the electron-electron correlation hole.
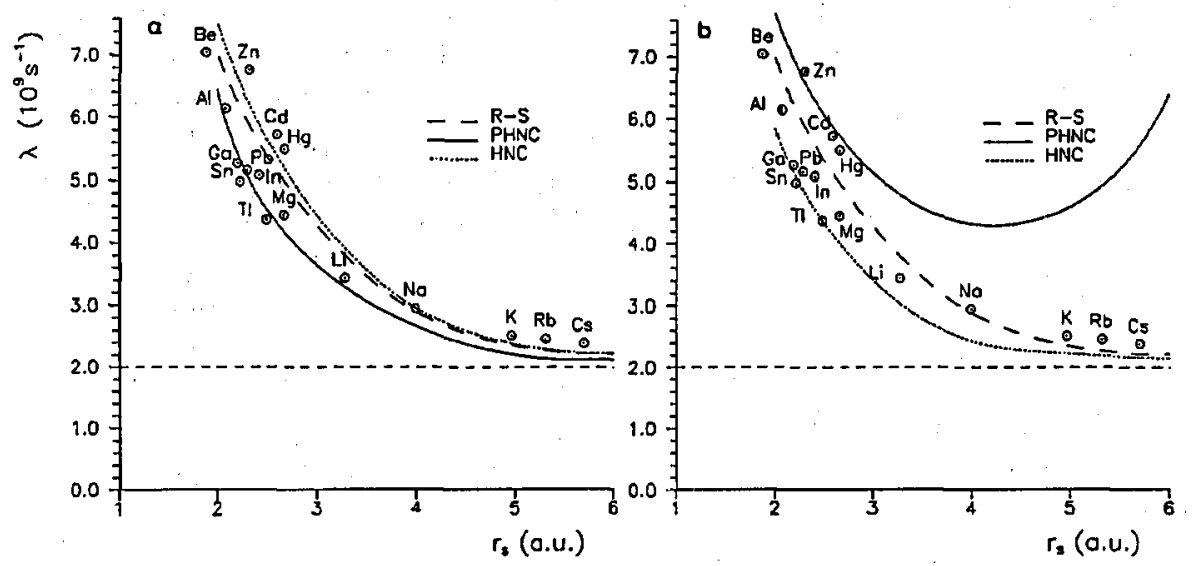

Fig. 2. Annihilation rates for different $\mathrm{e}^{+}-\mathrm{e}^{-}$potentials vs. $\boldsymbol{r}_{\mathbf{s}}$ : (a) from the formalisms in which the potentials have been determined, (b) obtained from the BG equation.

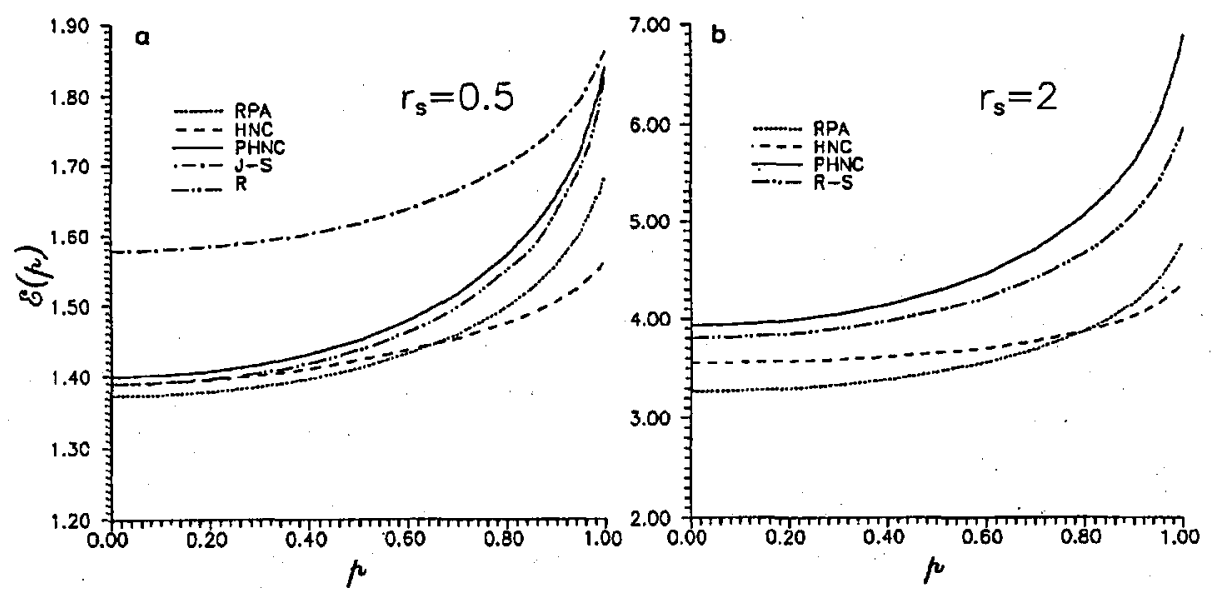

Fig. 3. Enhancement factors as functions of momentum $p$ for $r_{\mathrm{s}}=0.5$ (a) and $r_{\mathrm{s}}=2$ (b). Label $R$ corresponds to the results of Ref. [3].

In Fig. 2 it is shown that the annihilation rates calculated in the BG formalism at metallic densities are quite different from those obtained with the same potential within the formalism for which it is appropriate. The HNC potential 
while applied in the BG equation seems to lead to better results than the PHNC potential.

The momentum dependent enhancement factors are shown in Fig. 3 for $r_{\mathrm{s}}=$ 0.5 and 2 .

\section{Conclusions}

In general the potentials used in the BG equation lead to different predictions concerning the annihilation characteristics than when used in the formalism in which they have been determined. In particular, the PHNC potential fails completely for $r_{\mathrm{s}}>2.5$, while the HNC potential leads to quite satisfactory results. On the other hand, for the lower values of $r_{\mathrm{s}}<2$ the PHNC potential gives reasonable results and probably could be used when applying BML-like equations to core electrons, while taking into account the local density. This point, however, needs more thorough investigations. Remark that the PHNC approach includes the effect of the non-orthogonality of the one-electron wave functions and in this regard differs from the BG formalism. On the other hand, unlike the HNC approximation, and similarly as the BG approach it includes the effect of the kinetic energy of the positron.

The Jarlborg-Singh potential when applied in the BG equation leads to results which differ much from those obtained for any other potential at any electron density.

\section{Acknowledgment}

This work was supported by the Comnuttee for Scientific Research under grant No. 203079101 .

\section{References}

[1] H. Stachowiak, A. Rubaszek, in: Positrons at Metallic Surfaces, Ed. A. Ishii, in series Solid State Phenomena, Vol. 28/^9, Trans. Tech. Publications, Aedermannsdorf 1993, p. 7.

[2] S. Kahana, Phys. Rev. 129, 1622 (1963).

[3] A. Rubaszek, H. Stachowiak, E. Boroński, Z. Szotek, Phys. Rev. B 30, 1490 (1984).

[4] A. Rubaszek, H. Stachowiak, Phys. Rev. B 38, 3846 (1988).

[5] H. Stachowiak, Phys. Rev. B 41, 12522 (1990).

[6] H. Stachowiak, J. Lach, in: Positron Annihilation, Eds. Zs. Kajcsos, Cs. Szeles, Mater. Sci. Forum, Trans. Tech. Publ., Aedermannsdorf 1992, p. 853.

[7] J.P. Carbotte, Phys. Rev. 144, 309 (1966); J.P. Carbotte, A. Salvadori, Phys. Rev. 162,290 (1967).

[8] H. Sormann, W. Puff, in: Positron Annihilation, Eds. P.C. Jain, R.M. Singru, K.P. Gopinathan, World Sci., Singapore 1985, p. 161; H. Sormann, Phys. Status Solidi B 142, K45 (1987); H. Sormann, in: Positron Annihilation, Eds. L. Dorikens-Vanpraet, M. Dorikens, D. Segers, World Sci., Singapore 1989, p. 272.

[9] E. Bonderup, J.U. Andersen, D.N. Lowy, Phys. Rev. B 20, 883 (1979).

[10] E. Boronski, T. Jarlborg, in: Positron Annihilation, Eds. Zs. Kajcsos, Cs. Szeles, Mater. Sci. Forum, Trans. Tech. Publ., Aedermannsdorf 1992, p. 607.

[11] J. Gondzik, H. Stachowiak, J. Phys. C 18, 5399 (1985).

[12] T. Jarlborg, A.K. Singh, Phys. Rev. B 36, 4660 (1987). 